\title{
NOTAS
}

\section{NUEVO REGISTRO DE LOCALIDAD PARA OSTEOPILUS VASTUS (ANURA: HYLIDAE) EN LA REPÚBLICA DOMINICANA}

\author{
Cristian Marte Pimentel
}

Museo Nacional de Historia Natural de Santo Domingo. Calle César Nicolás Penson, Plaza de la Cultura, Santo Domingo, República Dominicana.c.marte@museohistorianatural.gov.do

\section{RESUMEN}

Se registra una nueva localidad para la rana arborícola gigante de La Hispaniola Osteopilus vastus (Cope, 1871) en Baní, provincia Peravia, República Dominicana.

Palabras clave: Osteopilus vastus, nuevo registro, República Dominicana, Hylidae.

Title: A new locality record for Osteopilus vastus (Anura: Hylidae) in the Dominican Republic.

\section{ABSTRACT}

A new locality is reported for the Hispaniolan Giant Treefrog Osteopilus vastus (Cope, 1871) in Baní, Peravia Province, Dominican Republic.

Key words: Osteopilus vastus, new record, Dominican Republic, Hylidae.

La especie de rana Osteopilus vastus fue descrita por Cope en 1871 bajo el nombre de Hyla vasta. Gracias a estudios con electroforesis proteica y secuenciación de ADN, en 2003, la especie fue ubicada en el género Osteopilus por Hedgges (1996). Actualmente, ésta se encuentra ampliamente distribuida en la isla (Schwartz y Henderson, 1991; Stuart et al., 2008; Fig. 1). Esta especie presenta un rango altitudinal que se extiende hasta los 1,697 sobre el nivel del mar (Stuart et al., 2008).

Osteopilus vastus es una de las ranas de La Hispaniola consideradas en peligro (EN) por la UICN (2011). El deterioro de sus hábitats, la reducción de la cobertura vegetal y el cambio en el uso de la tierra, fundamentalmente para actividades agrícolas, además de la distribución restringida, son los principales argumentos en que se apoya su consideración como una especie en peligro (Stuart et al., 2008).

La nueva localidad que se registra para $O$. vastus se encuentra en el extremo oriental de la Cordillera Central, en la vertiente suroeste de loma Los Guayuyos: $18^{\circ} 24^{\prime} 57.13^{\prime \prime} \mathrm{N}$; $70^{\circ} 23^{\prime}$ 16.20" O, 1,362 msnm., al sur del Parque Nacional Luis Quinn. El área se encuentra a unos 500 metros del cuerpo de agua más cercano, el nacimiento del Arroyo Bahía. La vegetación de la zona es un pastizal con pequeños parches de bosque latifoliado, perturbado por la actividad agrícola, principalmente la siembra de café (Coffea sp.) y guineos (Mussa sp.), aunque permanecen elementos del bosque original como árboles de cedro. El ejemplar fue encontrado durante búsquedas nocturnas, el 8 de enero de 2010 por el biólogo Robert Ortiz, como parte de un inventario rápido de la biodiversidad de la región, y se encuentra depositado en la colección herpetológica del Museo Nacional de Historia Natural de Santo Domingo (MNHNSD 23.744). 
Este nuevo registro se convierte en una de las localidades más al sur que presenta la distribución actual de la especie (Fig. 1). Resulta de interés registrar nuevas localidades fuera de su distribución conocida, ya que informaciones como esta pueden ser utilizadas para evaluaciones futuras del estado de conservación de la misma. Este hallazgo resalta la necesidad de realizar expediciones hacia nuevas localidades con características ecológicas similares (altura, vegetación, disponibilidad de cuerpos de agua) a las ya reconocidas como preferidas por O. vastus.

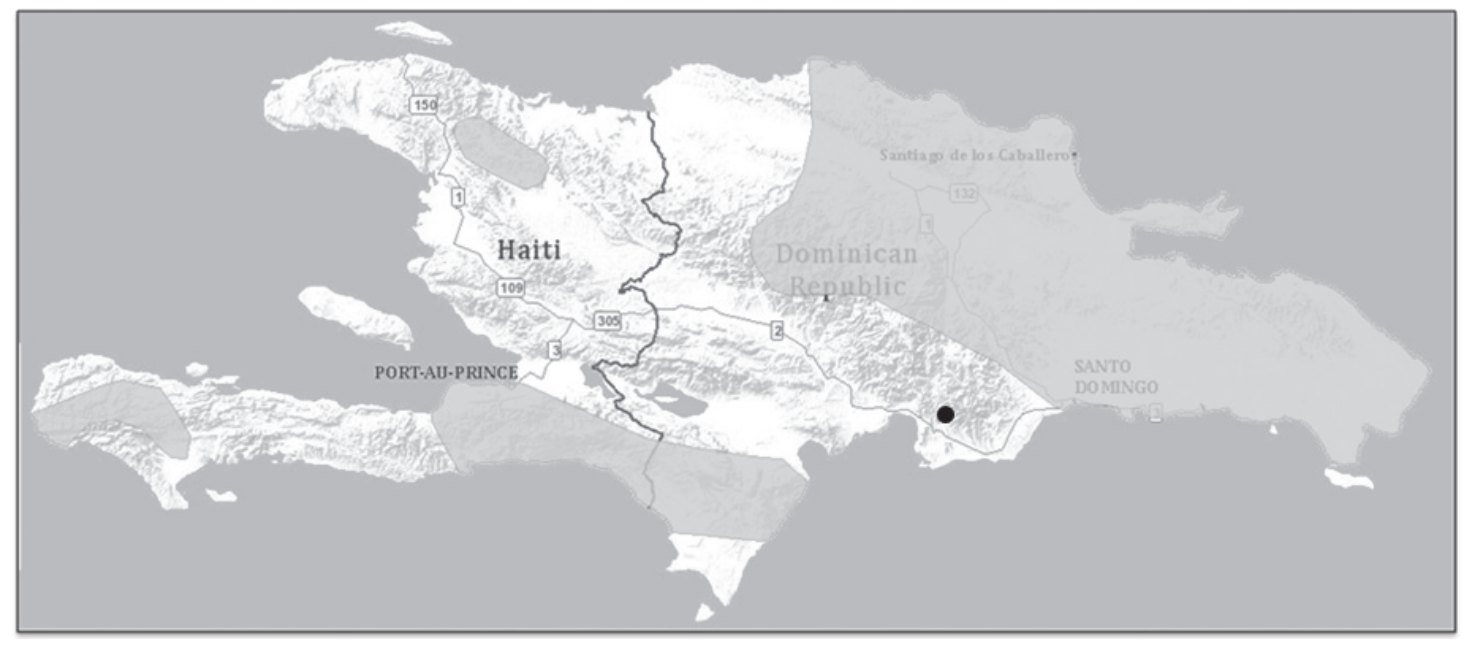

Figura 1, Distribución actual de Osteopilus vastus en La Hispaniola (IUCN Red List of Threatened Species, 2011.2). El punto negro representa el nuevo registro de localidad.

\section{AGRADECIMIENTOS}

Los resultados de esta nota se obtuvieron bajo el proyecto "Diversidad biológica de un área natural no protegida: Honduras-El Matadero, prov. Peravia. Propuesta para la valoración y aprovechamiento sostenible de sus recursos naturales", financiado por el Ministerio de Educación Superior, Ciencia y Tecnología (FONDOCYT, \# 2008-2-C2-040). Gracias a la colaboración del Instituto de Investigaciones Botánicas y Zoológicas de la Universidad Autónoma de Santo Domingo y al Museo Nacional de Historia Natural de Santo Domingo durante la ejecución de este proyecto.

\section{LITERATURA CITADA}

Hedges, S. B. 1996. The origin of West Indian amphibians and reptiles, p. 95-128. In R. Powell and R.W. Henderson (eds.), Contributions to West Indian Herpetology: A Tribute to Albert Schwartz. SSAR Contrib. Herpetol., vol. 12. Ithaca, New York.

IUCN 2011. IUCN Red List of Threatened Species. Version 2011.2.<www.iucnredlist.org>. Downloaded on 26 March 2012.

Schwartz, A. y Henderson R. W., 1991, Amphibian and Reptiles of the West Indies: descriptions, Distributions, and Natural History. University of Florida Press. Gainesville. 1-720 pp.

Stuart, S. N., M. Hoffmann, J. S. Chanson, N. A. Cox, R. J. Berridge, P. Ramani y B. E. Yong (eds) 2008. Threatened Amphibians of the World. Lynx Edicions, Barcelona, Spain; UICN, Gland, Switzerland; and Conservation International, Arlingtong, Virginia, USA. 1-758 pp.

[Recibido: 15 de marzo, 2012. Aceptado para publicación: 16 de mayo, 2012] 